\title{
Modelamiento y simulación del proceso de esterificación del ácido acético con metanol en una configuración compleja
}

Modeling and simulation of the acetic acid sterification process with methanol in a complex configuration

Recibido: noviembre 15 de 2019 | Revisado: diciembre 08 de 2019 | Aceptado: enero 18 de 2020

\author{
Luis Carrasco Venegas ${ }^{\mathrm{I}}$ \\ Luz Castañeda Pérez ${ }^{2}$
}

1 Unidad de Investigación de la Facultad de Ingeniería Química, Facultad de Ingeniería Química, Universidad Nacional del Callao, Callao, Perú.

2 Red Internacional de I+D+i+e, Escuela Universitaria de Posgrado, Universidad Nacional Federico Villarreal, Lima

\begin{abstract}
ResUMEN
Se investiga la dinámica del proceso de esterificación del ácido acético con metanol para la obtención de acetato de metilo, considerando el flujo de ácido acético desde un tanque cónico y el de metanol desde un tanque cilíndrico. En ambos casos, los flujos y la temperatura son variables. Los reactivos se descargan a un reactor esférico, donde se realiza la reacción de esterificación, las condiciones de volumen, temperatura y concentración son dependientes del tiempo. La constante específica de velocidad de reacción y la constante de equilibrio en función a la temperatura son datos reportados de literatura especializada. Para el modelamiento de este proceso se establece los balances de masa global, balance de masa por cada componente y los balances de energía en cada uno de los tanques lo cual conduce a la obtención de un sistema de catorce ecuaciones diferenciales ordinarias resueltas a través del software Polymath. Se obtienen los perfiles de temperatura y concentración en cada uno de los tanques y el reactor, asimismo, la altura y volumen de fluido en cada uno delos tanques. Los resultados dependen de las condiciones de alimentación de la mezcla reaccionante y de las condiciones mantenidas en el reactor.
\end{abstract}

Palabras clave: modelamiento, simulación, dinámica, destilación reactiva, acetato de metilo, configuración compleja

\begin{abstract}
The dynamics of the process of sterification of acetic acid with methanol in order to obtain methyl acetate have been studied, considering the flow of acetic acid from a conical tank and that of methanol from a cylindrical tank. In both cases, the flows and temperature are variable. The reagents are discharged to a spherical reactor, where the esterification reaction occurs, and where the conditions of volume, temperature and concentration are time dependent. The specific reaction rate constant and the equilibrium constant as a function of temperature are reported data obtained from specialized literature. For the modeling of this process, the global mass balances, mass balance for each component and the energy balances in each of the conical, cylindrical and spherical tanks are established, which results in a system of fourteen ordinary differential equations which have been resolved using the Polymath software. Temperature and concentration profiles are measured in each of the tanks and the reactor; as well as the height and volume of fluid in each of the tanks. The results depend on the feed conditions of the reaction mixture and the conditions maintained in the reactor. The modeling and simulation of complex processes constitute an important tool for the prediction of possible favorable results for the operating conditions.
\end{abstract}

Key words: modeling, simulation, dynamics, reactive distillation, methyl acetate, complex configuration

(C) Los autores. Este artículo es publicado por la Revista Campus de la Facultad de Ingeniería y Arquitectura de la Universidad de San Martín de Porres. Este artículo se distribuye en los términos de la Licencia Creative Commons Atribución No-comercial - Compartir-Igual 4.0 Internacional (https://creativecommons.org/licenses/ CC-BY), que permite el uso no comercial, distribución y reproducción en cualquier medio siempre que la obra original sea debidamente citada. Para uso comercial contactar a: revistacampus@usmp.pe. 


\section{Introducción}

La dinámica de los procesos de reacción ha sido poco estudiada debido a la relativa complejidad que involucra su desarrollo analítico y experimental; sin embargo, un correcto planteamiento de las ecuaciones de conservación de materia y energía facilita el planteamiento de los modelos. Grau, D. (1999) realiza un estudio de tallado de la operación de los reactores batch, continuo y semicontinuo mediante la formulación de las ecuaciones de conservación respectivas; sin embargo, no toma en cuenta el flujo variable a la entrada del reactor. El estudio de reactores por semilotes, conocido como semicontinuo ha sido desarrollado por Fogler (2001), aplicando el balance de materia al proceso de esterificación del ácido acético con etanol; sin embargo, este proceso ha sido desarrollado para condiciones isotérmicas, con retiro parcial de acetato de etilo. Aldas, J., Vivar, G. (2014), en su investigación "Diseño y construcción de un reactor semicontinuo para la obtención de acetato de sodio" realizó la construcción de un reactor semicontinuo basándose en la ecuación diferencial lineal para el diseńo del reactor y mediante ensayos de laboratorio determinó las variables de operación del sistema. Ovando, V. (2007) en su investigación "Estudio teórico y experimental de la copolimerizacion de acetato de vinilo y acrilato de butilo en microemulsiones estabilizadas aniónica y estéricamente en procesos por lotes y semicontinuos" presenta los balances de materia de este proceso y realiza pruebas experimentales.

Elestudiodelos reactores semicontinuo se aborda en Cutlip, M. y Shacham, M. (2008) que hace un estudio parcial de los reactores semicontinuos aplicado a la destilación reactiva del acetato de etilo usando una configuración clásica y reacción química y destilación simultánea. Lux (2013) y Rohde (2004) han realizado estudios de esterificación del ácido acético con metanol usando resinas como catalizadores sólidos, en reactores por lotes. En ese contexto es importante decir que el modelamiento de procesos semicontinuo fue desarrollado por Carrasco (2018) quien planteó los balances de materia y energía de sistemas semicontinuos complejos. Auquilla, Carrasco (2017) desarrollaron la investigación denominada: "Modelamiento y simulación del proceso de obtención del acetato de etilo en un reactor esférico"; (Guerrero, Carrasco, 2017) en su investigación sobre reactores semicontinuos desarrollaron el trabajo denominado: "Modelamiento y simulación del proceso de obtención del acetato de metilo en un reactor esférico". En ambos casos, fue utilizado un reactor de vidrio que está compuesto de dos depósitos uno cilíndrico y otro cónico; la descarga va a un tanque esférico (reactor), donde se produce la reacción de esterificación, obteniéndose una mezcla de productos formados y reactantes no consumidos. Adicionalmente, la constante de velocidad de reacción y la constante de equilibrio fueron tomados de Howard (1997), Huss (2003) y Lux (2013), las propiedades termodinámicas se encuentran en Perry's Chemicals Engineering Handbook (1984), Ullman's Encyclopediatrial Chemistry (1985) y CRC Handbook of Chemistry and Physics (1992).

En este trabajo, se presenta el modelamiento de un sistema semicontinuo complejo. Como base 
de estudio se ha tomado el proceso de esterificación del ácido acético con metanol para la obtención de acetato de metilo y como sub producto el agua. El acetato de metilo tiene la característica de ser el componente más volátil de las cuatro sustancias que intervienen en el proceso por lo que es posible separar este compuesto conforme se va formando, proceso conocido como destilación reactiva. Como resultado del modelamiento, se obtiene un sistema de ecuaciones diferenciales ordinarias no homogéneas los cuales son resueltas por uno de los métodos explícitos de resolución de ecuaciones diferenciales de valor inicial. Se requiere el conocimiento de las propiedades físico químicas de cada uno de los componentes de este sistema de esterificación, así como las propiedades del sistema como la entalpia de reacción, la constante cinética y la constante de equilibrio, así como las condiciones iniciales.

\section{Método}

Se utiliza el método de prueba y error para la calibración del modelo en cuanto se refiere a las condiciones iniciales de ingreso al reactor esférico; una vez que se logra la convergencia, se procede a simular otros casos de estudio, como los que se presenta en este trabajo.

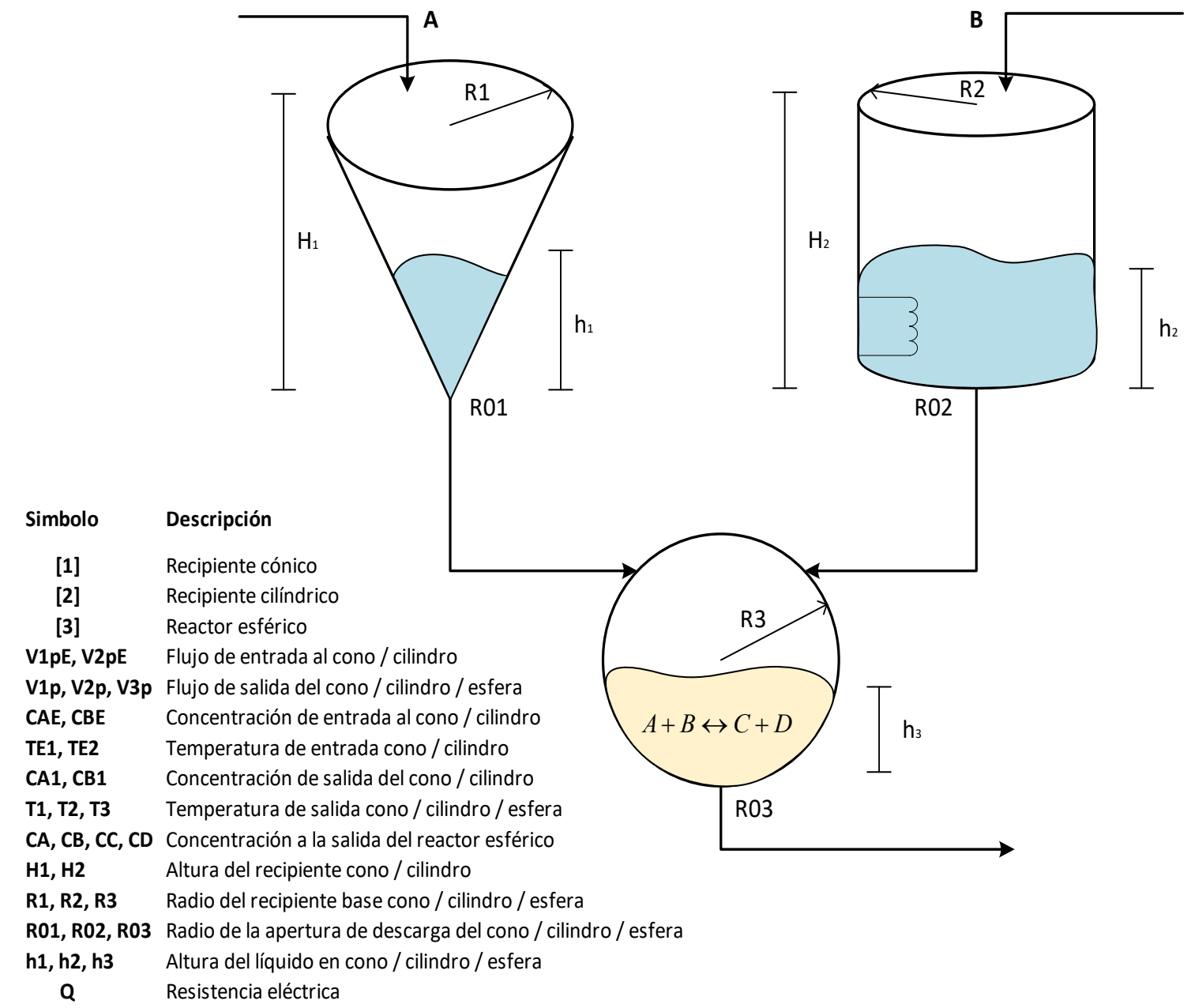

Figura 1. Esquema del sistema complejo de proceso de esterificación del ácido acético con metanol 


\section{Procedimiento}

Se plantea las ecuaciones diferenciales proveniente de los balances de materia total, de los balances de materia por componente y los balances de energía aplicado a cada uno de los recipientes mostrados en la Figura 1. Posteriormente se resuelve dichas ecuaciones con la ayuda del software polymath, que permite encontrar las variaciones temporales de volumen, altura del fluido, concentracion y temperatura en cada uno de los tanques.

La reacción que se lleva a cabo en este sistema es la esterificación del ácido acético con metanol dada por:

$$
\begin{gathered}
\mathrm{CH}_{3} \mathrm{COOH}+\mathrm{CH}_{3} \mathrm{OH} \underset{k 2}{\stackrel{k 1}{\rightleftarrows}} \mathrm{H}_{2} \mathrm{O}+\mathrm{CH}_{3} \mathrm{COOCH}_{3} \\
A+B \underset{k 2}{\stackrel{k 1}{\rightleftarrows}} \mathrm{C}+D
\end{gathered}
$$

\section{Balance de masa total en el tanque cónico}

La tasa de acumulación es igual al flujo neto de entrada al reactor, es decir:

$$
\frac{\partial}{\partial t} \iiint \rho d v=-\iint \rho(\underline{V} \cdot \bar{n}) d A
$$

Consideremos que la densidad del fluido es constante, con lo cual, la ecuación (1) se transforma en:

$$
\frac{d v_{1}}{d t}=v_{1 E}^{\circ}-v_{1}
$$

El flujo volumétrico a la salida del tanque cónico se obtiene mediante la ecuación de Torricelli, la cual es:

$$
{\stackrel{\circ}{v_{1}}}^{\prime}=\pi R_{01}^{2} \sqrt{2 g_{1}}
$$

El volumen instantáneo en el tanque cónico se obtiene mediante:

$$
v_{1}=\frac{\pi}{3} r^{2} \times h_{1}
$$

Para expresar el radio instantáneo en términos de la altura instantánea se usa la semejanza de triángulos, dada por:

$$
\frac{r}{h_{1}}=\frac{R_{1}}{H_{1}}
$$

De la ecuación (5), se tiene:

$$
r^{2}=\frac{R_{1}^{2} \times h_{1}^{2}}{H_{1}^{2}}
$$

Reemplazando (6) en (4)

$$
v_{1}=\frac{\pi}{3}\left(\frac{R_{1}}{H_{1}}\right)^{2} \times h_{1}^{3}
$$

Derivando la ecuación (7), se tiene:

$$
\frac{d v_{1}}{d t}=\pi\left(\frac{R_{1}}{H_{1}}\right)^{2} \times h_{1}^{2} \times \frac{d h_{1}}{d t}
$$

Igualando las ecuaciones (8) y (2)

$$
\begin{aligned}
\frac{d h_{1}}{d t} & =\frac{1}{\pi}\left(\frac{H_{1}}{h_{1}}\right)^{2} \times \frac{1}{R_{1}^{2}}\left(\dot{\circ}_{v_{1}}-\dot{\circ}_{2}\right) \\
h_{1}(U) & =h_{10}
\end{aligned}
$$

\section{Balance de materia del componente A en el tanque cónico}

La acumulación del componente $\mathrm{A}$ en el tanque cónico es igual a la tasa neta de flujo molar de A mas la tasa de generación de A.

$\frac{\partial}{\partial t} \iiint C_{A} d v=-\iint C_{A}(\underline{V} \cdot \bar{n}) d_{A}+\iiint r_{A} d v$

Dado que no hay reacción química en el tanque de almacenamiento cónico, el término de generación es igual a cero, por lo cual, la ecuación (10) se transforma en:

$$
\frac{\partial}{\partial t}\left(C_{A 1} v_{1}\right)=\dot{\circ}_{1} C_{A E}-\dot{\circ}_{2} C_{A 1}
$$

Dado que en este caso varían tanto la concentración como el volumen:

$$
v_{1} \frac{d C_{A 1}}{d t}+C_{A 1} \frac{d v_{1}}{d t}=\dot{\circ}_{1} C_{A E}-\dot{\circ}_{2} C_{A 1}
$$

Reemplazando (2) en (12), se tiene: 
$v_{1} \frac{d C_{A 1}}{d t}=-C_{A 1}\left(\dot{\circ}_{1}-\dot{\circ}_{2}\right)+\dot{\circ}_{1} C_{A E}-\dot{\circ}_{2} C_{A 1}$

Simplificando la ecuación (13)

$$
\frac{d C_{A 1}}{d t}=\frac{\dot{v}_{1}}{v_{1}}\left(C_{A E}-C_{A 1}\right)
$$

La ecuación (5.14) se resuelve con la condición inicial: $\mathrm{C}_{\mathrm{A} 1}(\mathrm{O})=\mathrm{C}_{\mathrm{AO}}$.

\section{Balance de energía en el tanque cónico}

La energía acumulada es igual al flujo neto de energía que contiene el fluido mas el flujo de energía generada menos la perdida de energía por intercambio al medio circundante.

$$
\frac{\partial}{\partial t} \iiint\left(\rho_{1} C p_{1} T_{1}\right) d v_{1}=-\iint \rho_{1} C p_{1} T_{1}(\underline{V} \cdot \bar{n}) d A+\iiint G d v_{1}-U_{1} A_{1}\left(T_{1}-T_{\infty 1}\right)
$$

En el tanque de almacenamiento no se produce reacción química, por la que el término generación se anula.

$$
\rho_{1} C p_{1} \frac{\partial}{\partial t}\left(v_{1} T_{1}\right)=\rho C p \dot{\circ}_{1 E} T_{E 1}-\rho C p \dot{\circ}_{1} T_{1}-U_{1} A_{1}\left(T_{1}-T_{\infty 1}\right)
$$

Simplificando la ecuación (16)

$$
\frac{\partial}{\partial t}\left(v_{1} T_{1}\right)=\stackrel{\circ}{v_{1 E}} T_{E 1}-\stackrel{\circ}{v}_{1} T_{1}-\frac{U_{1} A_{1}}{\rho_{1} C p_{1}}\left(T_{1}-T_{\infty 1}\right)
$$

En la ecuación (17) tanto el volumen por lo cual se tiene: como la concentración, son variables,

$$
v_{1} \frac{\partial T_{1}}{\partial t}+T_{1} \frac{\partial v_{1}}{\partial t}=\dot{v}_{1 E} T_{E 1}-\dot{v}_{1} T_{1}-\frac{U_{1} A_{1}}{\rho_{1} C p_{1}}\left(T_{1}-T_{\infty 1}\right)
$$

Reemplazando la ecuación (2) en (18) y simplificando, se obtiene:

$$
\frac{\partial T_{1}}{\partial t}=v_{1 E}^{\circ} \frac{\left(T_{E 1}-T_{1}\right)}{v_{1}}-\frac{U_{1} A_{1}}{\rho_{1} C p_{1} v_{1}}\left(T_{1}-T_{\infty 1}\right)
$$

La condición inicial para la resolución de (19) es: $T_{1}(0)=T_{10}$

\section{Balance de materia global en el tanque cilíndrico}

La masa acumulada en el tanque cilíndrico es igual al flujo neto de materia en dicho tanque

$$
\frac{\partial}{\partial \mathrm{t}} \iiint \rho d v_{2}=-\iint \rho(\underline{V} \cdot \bar{n}) d A
$$

De la ecuación (20) para $\rho$ constante, se tiene:

$$
\frac{d v_{2}}{d t}=\dot{v}_{2 E}^{\circ}-\dot{v}_{2}
$$

De la ecuación de Torricelli, se obtiene

$$
\stackrel{\circ}{v}_{2}=\pi R_{02}^{2} \sqrt{2 g h_{2}}
$$

El volumen instantáneo se obtiene mediante

$$
v_{2}=\pi R_{2}^{2} h_{2}
$$

Derivando la ecuación (23)

$$
\frac{d v_{2}}{d t}=\pi R_{2}^{2} \frac{d h_{2}}{d t}
$$

Igualando las ecuaciones (21) y (24), se obtiene:

$$
\frac{d h_{2}}{d t}=\frac{1}{\pi R_{2}^{2}}\left(\dot{0}_{2 E}^{\circ}-\dot{v}_{2}\right)
$$

\section{Balance de materia del componente $B$ en el tanque cilíndrico}

La acumulación del componente B en 
el tanque cilíndrico es igual al flujo neto en el tanque mas la tasa de generación.

$$
\frac{\partial}{\partial t} \iiint C_{B 1} d v_{2}=-\iint C_{B 1}(\underline{V} \cdot \bar{n}) d A+\iiint r_{B} d v_{2}
$$

En el tanque cilíndrico, no se produce reacción química alguna, por lo cual, en la ecuación (26) se anula el término de generación de materia, luego al simplificar, resulta:

$$
\frac{\partial}{\partial t}\left(C_{B 1} v_{2}\right)=v_{2 E}^{\circ} C_{B E}-\dot{v}_{2} C_{B 1}
$$

Considerando que el volumen y la concentración varía con el tiempo, derivando la ecuación (27) con la ayuda de la ecuación (21) y simplificado se tiene:

$$
\frac{d C_{B 1}}{d t}=\frac{\stackrel{\circ}{v}_{2}}{v_{2}}\left(C_{B E}-C_{B 1}\right)
$$

La ecuación (28) requiere la condición inicial $C_{B 1}(0)=C_{B 0}$

\section{Balance de energía en el tanque cilíndrico}

La tasa de acumulación de energía en el tanque cilíndrico es igual al flujo neto de anergia debido al flujo más el flujo de calor añadido al tanque, menos la pérdida de energía al medio circundante.

$$
\frac{\partial}{\partial t} \iiint\left(\rho_{2} C p_{2} T_{2}\right) d v_{2}=-\oint \int \rho_{2} C p_{2} T_{2}(\underline{V} \cdot \bar{n}) d_{A}+Q-U_{2} A_{2}\left(T_{2}-T_{\infty 2}\right)
$$

Para $\rho$ y Cp constantes, la ecuación (29), se transforma en:

$$
\rho_{2} C p_{2} \frac{\partial}{\partial t}\left(v_{2} T_{2}\right)=\rho_{2} C p_{2} v_{2 E}^{\circ} T_{E 2}-\rho_{2} C p_{2} \dot{v}_{2} T_{2}+Q-U_{2} A_{2}\left(T_{2}-T_{\infty 2}\right)
$$

Expandiendo la ecuación (30)

$$
T_{2} \frac{\partial v_{2}}{\partial t}+v_{2} \frac{\partial T_{2}}{\partial t}=\dot{v}_{2 E} T_{E 2}-\dot{v}_{2} T_{2}+\frac{Q}{\rho_{2} C p_{2}}-\frac{U_{2} A_{2}}{\rho_{2} C p_{2}}\left(T_{2}-T_{\infty 2}\right)
$$

Con la ayuda de la ecuación (21), la ecuación (31) resulta:

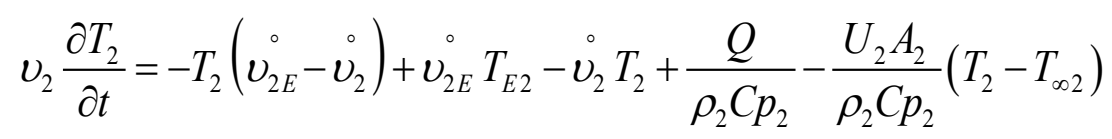

Simplificando la ecuación (32)

$$
\frac{d T_{2}}{d t}=\frac{v_{2 E}^{\circ}}{v_{2}}\left(T_{E 2}-T_{2}\right)+\frac{Q}{\rho_{2} C p_{2} v_{2}}-\frac{U_{2} A_{2}}{\rho_{2} C p_{2} v_{2}}\left(T_{2}-T_{\infty 2}\right)
$$

La ecuación (33) se resuelve con la condición inicial $T_{2}(0)=T_{20}$

\section{Balance de masa global en el reactor esférico}

Al reactor esférico ingresa el flujo volumétrico de ácido acético y el flujo volumétrico de metanol y a la salida se tiene los flujos de la mezcla reaccionante en fase líquida, por tanto, la tasa de acumulación viene dada por:

$$
\frac{d v_{3}}{d t}=\stackrel{\circ}{v_{1}}+\stackrel{\circ}{v_{2}}-\stackrel{\circ}{v_{3}}
$$


El flujo volumétrico a la salida del tanque esférico, está dado por la ecuación de Torricelli.

$$
{\stackrel{\circ}{v_{3}}}^{\prime}=\pi R_{03}^{2} \sqrt{2 \times g \times h_{3}}
$$

El volumen instantáneo en el reactor esférico en función a la altura se encuentra usando el concepto de rotación de un plano cuya ecuación es

$$
v_{3}=\pi \int_{0}^{h}[f(y)]^{2} d y
$$

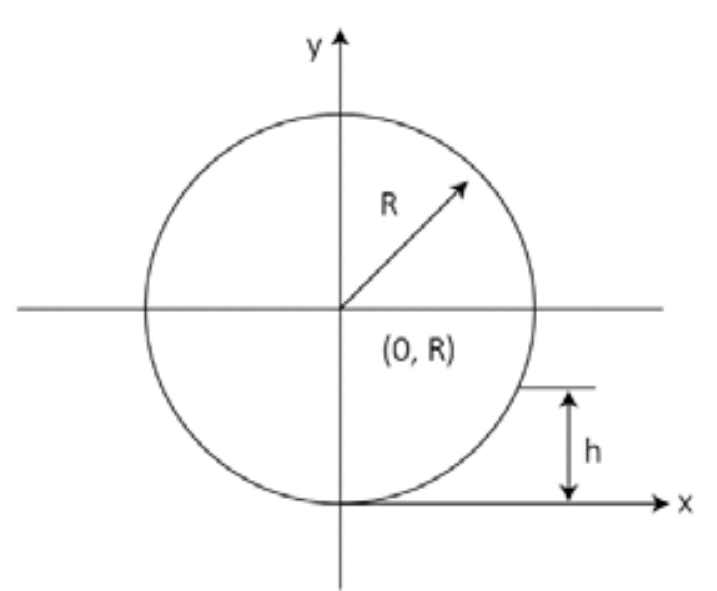

Figura 2. Esquema para el cálculo del volumen instantáneo.

La ecuación del círculo con centro ( $\mathrm{x}$, $y)=(0, R)$ está dado por:

$$
x^{2}+(y-R)^{2}=R^{2}
$$

A partir de la ecuación (37) se tiene:

$$
x=\sqrt{R^{2}-(y-R)^{2}}=\sqrt{2 R y-y^{2}}=f(y)
$$

Reemplazando (38) en (36)

$$
v_{3}=\pi \int_{0}^{h}\left(\sqrt{2 R y-y^{2}}\right)^{2} d y
$$

La integración de la ecuación (39) conduce a obtener el volumen instantáneo de mezcla reaccionante

$$
v_{3}=\pi\left(R h_{3}^{2}-\frac{h_{3}^{3}}{3}\right)
$$

Derivando respecto al tiempo

$$
\frac{d v_{3}}{d t}=\left(2 \pi R h_{3}-\pi h_{3}^{2}\right) \frac{d h_{3}}{d t}
$$

Igualando las ecuaciones (34) y (41)

$$
\frac{d h_{3}}{d t}=\frac{\left(\begin{array}{c}
\circ \\
\dot{v}_{1}+\dot{v}_{2}-\circ_{3}
\end{array}\right)}{2 \pi R h_{3}-\pi h_{3}^{2}}
$$

La ecuación (42) requiere la siguiente condición:

$$
t=0, h_{3}(0)=h_{30}
$$

\section{Balance de materia de acido acético en el reactor}

La tasa de acumulación de $\mathrm{A}$ en el reactor esférico viene dada por la diferencia entre los flujos de ingreso y salida mas la tasa de generación.

$$
\frac{d C_{A}}{d t}=\frac{\dot{v}_{1} \times C_{A 1}-\left(\dot{v}_{1}+\dot{v}_{2}\right) \times C_{A}}{v_{3}}+r_{A}
$$

La ecuación (43) requiere la condición inicial: $C_{A}(0)=C_{A 0}$

\section{Balance de materia de metanol en el reactor}

La tasa de acumulación de $\mathrm{B}$ en el reactor esférico viene dada por la diferencia entre los flujos de ingreso y salida más la tasa de generación.

$$
\frac{d C_{B}}{d t}=\frac{\dot{v}_{1} \times C_{B 1}-\left(\dot{v}_{1}+\dot{v}_{2}\right) \times C_{B}}{v_{3}}+r_{B}
$$

La ecuación (44) requiere la condición inicial: $C_{B}(0)=C_{B 0}$

\section{Balance de materia de acetato de metilo en el reactor}

La tasa de acumulación de $\mathrm{D}$ en el reactor esférico viene dada por el flujo de salida más la tasa de generación por reacción. 


$$
\frac{d C_{D}}{d t}=-\frac{\left(\dot{v}_{1}+\dot{v}_{2}\right) \times C_{D}}{v_{3}}+r_{D}
$$

La ecuación (45) requiere la condición inicial: $C_{D}(0)=0$

\section{Balance de materia de acetato de metilo en el reactor}

La tasa de acumulación de $\mathrm{C}$ en el reactor esférico viene dada por el flujo de salida más la tasa de generación por reacción.

$$
\frac{d C_{C}}{d t}=-\frac{\left(\dot{v}_{1}+\dot{v}_{2}\right) \times C_{C}}{v_{3}}+r_{C}
$$

La ecuación (46) requiere la condición inicial:

$$
\begin{aligned}
& C_{C}(0)=C_{C 0} \\
& \frac{\partial}{\partial t} \iiint\left(\rho \mathrm{CpT}_{3}\right) d v_{3}=-\iint \rho C p T_{3}(\underline{V} \cdot \bar{n}) d A+\iiint G_{d v_{3}}-U_{3} A_{3}\left(T_{3}-T_{\infty 3}\right)
\end{aligned}
$$

Considerando que las propiedades físicas como el calor específico y la
La velocidad de reacción del ácido acético está dada por:

$$
r_{A}=-k_{1} C_{A} C_{B}+k_{2} C_{c} C_{D}
$$

La relación de velocidades de reacción esta dado por

$$
-r_{A}=-r_{B}=r_{C}=r_{D}
$$

\section{Balance de energía en el reactor esférico}

La tasa de acumulación de energía en el reactor esférico es igual al flujo neto de energía en el tanque más la tasa de generación de energía mas la tasa de intercambio de calor con el medio

$$
\rho \operatorname{Cp} \frac{\partial}{\partial \mathrm{t}}\left(T_{3} \mathrm{v}_{3}\right)=\rho \operatorname{Cp} \dot{0}_{1} T_{1}+\rho \operatorname{Cp} \dot{0}_{2} T_{2}-\rho C p \dot{0}_{3} T_{3}+G v_{3}-U_{3} A_{3}\left(T_{3}-T_{\infty 3}\right)
$$

Dividiendo entre $\rho C p$, se obtiene

$$
\frac{\partial}{\partial t}\left(T_{3} v_{3}\right)=\stackrel{\circ}{v_{1}} T_{1}+\stackrel{\circ}{v}_{2} T_{2}-\dot{0}_{3} T_{3}-\dot{\circ}_{4} T_{3}+\frac{G v_{3}}{\rho C p}-\frac{U_{3} A_{3}}{\rho C p}\left(T_{3}-T_{\infty 3}\right)
$$

Teniendo en consideración la ecuación (5.34) y simplificando, se tiene:

$$
\frac{d T_{3}}{d t}=\frac{\dot{\circ}_{1}\left(T_{1}-T_{3}\right)+\dot{\circ}_{2}\left(T_{2}-T_{3}\right)}{v_{3}}+\frac{G}{\rho C p}-\frac{U_{3} A_{3}}{\rho C p v_{3}}\left(T_{3}-T_{\infty 3}\right)
$$

La tasa de generación de energía se obtiene multiplicando la entalpia de reacción y la velocidad de reacción, es decir:

$$
G=\Delta H_{R} \times r_{A}
$$

La entalpia de reacción se obtiene mediante la diferencia de la entalpia de formación de productos y reactantes.

$$
\Delta H_{R}=\sum n_{i} H_{f i}(T)_{p}-\sum n_{i} H_{f i}(T)_{R}
$$

Para los componentes del sistema de estudio: acido acético, metanol, acetato de metilo y agua, las entalpias de formación en $\mathrm{Kj} / \mathrm{mol}$, indicados en CRC Handbook of Chemistry and Physics (1992), Perry's Chemical Engineering Handbook (1984), son:

$$
\begin{aligned}
& H_{f A}(T)=-422.584-4.8354 \times 10^{-2} T+2.3337 \times 10^{-5} T^{2} \quad(55) \\
& H_{f B}(T)=-188.188-4.9823 \times 10^{-2} T+2.0791 \times 10^{-5} T^{2}(56) \\
& H_{f D}(T)=-392.226-7.0347 \times 10^{-2} T+3.4601 \times 10^{-5} T^{2}(57)
\end{aligned}
$$




$$
H_{f D}(T)=-241.80
$$

El flujo molar de cada uno de los componentes se obtiene multiplicando la concentración por el flujo volumétrico a la salida.

$$
\begin{aligned}
& F_{A L}=C_{A} \times v_{3} \\
& F_{B L}=C_{B} \times v_{3} \\
& F_{C L}=C_{C} \times v_{3} \\
& F_{D L}=C_{D} \times v_{3}
\end{aligned}
$$

El número de moles instantáneo de cada componente en el reactor esférico, está dado por:

$$
\begin{aligned}
& N_{A}=C_{A} \times v_{3} \\
& N_{B}=C_{B} \times v_{3} \\
& N_{C}=C_{C} \times v_{3} \\
& N_{D}=C_{D} \times v_{3}
\end{aligned}
$$

El número total de moles en el reactor se obtiene sumando las ecuaciones

$$
N_{T}=N_{A}+N_{B}+N_{C}+N_{D}
$$

La composición molar de cada componente dentro del reactor esférico para cada componente se obtiene:

$$
Y_{i}=\frac{N_{i}}{N_{T}}
$$

El programa utilizado para la resolución del sistema de ecuaciones diferenciales obtenidas, se muestra a continuación.

\# 1.-Balance de masa global en el tanque cónico

$d(b 1) / d(t)=\boldsymbol{I f}(h 1<H 1)$ Then $((1 / p i)$ * $(H 1 / h 1) \wedge 2$ * $(1 / R 1 \wedge 2) *(V 1 p E-$ V1p)) Else (0)

$$
\begin{aligned}
& d(V 1) / d(t)=(V 1 p E-V 1 p) \\
& V 1 p=p i{ }^{*} R 01 \wedge 2^{*}\left(2^{*} g^{*} h 1\right) \wedge 0.5 \\
& V 1 \_I N=(1 / 3)^{*} p i^{*}(R 1 / H 1) \wedge 2^{*} h 1 \\
& \wedge 3 V 1(0)=0.1206374 \\
& h 1(0)=2 \\
& h 10=2 \\
& p i=3.1416 \\
& g=9.8 \\
& H 1=2.5 \\
& R 1=0.3 \\
& V 1 p E=0.0011 \\
& R 01=0.0095
\end{aligned}
$$

\# 2.- Balance de materia de A en el tanque cónico

$d(C A 1) / d(t)=(V 1 p / V 1) *(C A E-C A 1)$

$C A 1(0)=0.2$

$C A E=0.4$

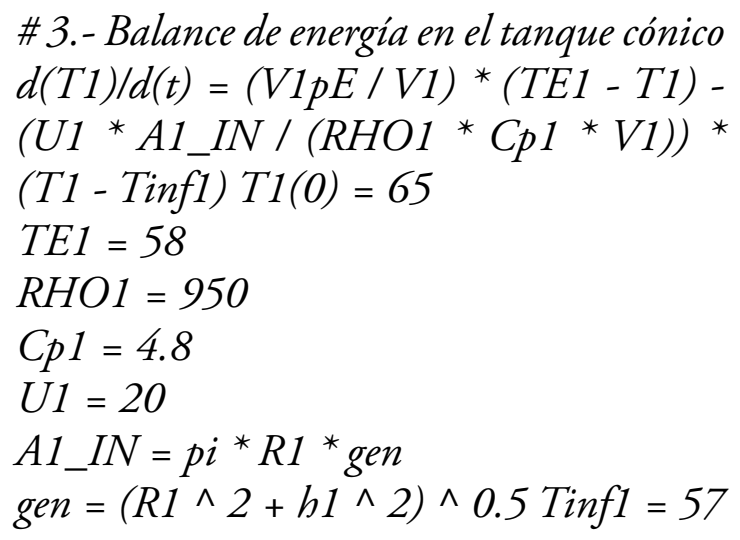

\# 4.- Balance de materia de masa global en el tanque cilindrico

$d(h 2) / d(t)=(1 /(p i * R 2 \wedge 2)) *(V 2 p E-$ $V 2 p) d(V 2) / d(t)=V 2 p E-V 2 p$

$h 2(0)=0.5$

$h 20=0.5$

$V 2(0)=1.5708$

$V 2 \_I N=p i{ }^{*} R 2 \wedge 2 * h 2 R 2=1$

$V 2 p E=0.0005$

$V 2 p=p i{ }^{*} R 02 \wedge 2{ }^{*}\left(2{ }^{*} g^{*} h 2\right) \wedge 0.5$ $R 02=0.008$

\# 5.- Balance de materia de B en el tanque cilindrico

$d(C B 1) / d(t)=(V 2 p / V 2) *(C B E-C B 1)$ 


$$
\begin{aligned}
& C B 1(0)=0.2 \\
& C B E=0.4 \\
& \text { \# 6.- Balance de energía en el tanque } \\
& \text { cilindrico } d(T 2) / d(t)=(V 2 p E / V 2) * \\
& (T E 2-T 2)+\left(Q /\left(\mathrm{RHO}_{2}{ }^{*} \mathrm{Cp} 2{ }^{*} V 2\right)\right)- \\
& \text { (U2 * A2_IN / (RHO2 * Cp2 } \\
& \left.{ }^{*} \text { V2) }\right)^{*}(T 2-\text { Tinf2) } T 2(0)=55 \\
& \text { TE2 }=45 \\
& \mathrm{RHO} 2=800 \\
& C p 2=4.533 \\
& U 2=30 \\
& A 2 \_I N=p i * R 2 \wedge 2 * h 2 \operatorname{Tinf} 2=40 \\
& Q=1000 \\
& V 3(0)=0.070774 \\
& V 3 \_I N=p i * h 3^{\wedge} 2^{*}\left(3^{*} R 3-h 3\right) \\
& V 3 p=p i{ }^{*} R 03 \wedge 2{ }^{*}\left(2{ }^{*} g^{*} h 3\right) \wedge 0.5 \\
& V 3 C=p i^{*}\left(R 3^{*} h 3 \wedge 2-h 3 \wedge 3 / 3\right) \\
& d(h 3) / d(t)=(V 1 p+V 2 p-V 3 p) /\left(2^{*} p i\right. \\
& { }^{*} \mathrm{R} 3{ }^{*} \mathrm{~h} 3 \\
& -p i^{*} h 3 \wedge \text { 2) } h 3(0)=0.08 \\
& h 30=0.08 \\
& R 3=1.2 \\
& R 03=0.01 \# 0.005 \# \# \text { \#0.015 cambio }
\end{aligned}
$$

\# 8.- Balance de masa de $A$ en el reactor $d(C A) / d(t)=\left(V 1 p{ }^{*} C A 1-(V 1 p+V 2 p)\right.$

$\left.{ }^{*} C A\right) / V 3$

$+r A$

$C A(0)=0.4$

\# 9. - Balance de materia de B

$d(C B) / d(t)=\left(V 2 p{ }^{*} C B 1-(V 1 p+V 2 p)\right.$

$\left.{ }^{*} C B\right) / V 3$

$+r B$

$C B(0)=0.4$

\# 10.- Balance de materia de $D$ en el reactor $d(C D) / d(t)=(-C D / V 3) *(V 1 p+$ $V 2 p)+r D C D(0)=0$
\# 11. - Balance de materia de $C$ en el reactor $d(C C) / d(t)=(-C C / V 3) *(V 1 p+V 2 p)+$ $r C C C(0)=0.0005$

\# 12.-Balance de energía en el reactor esférico $d(T 3) / d(t)=(V 1 p *(T 1-T 3)$ $\left.+V 2 p{ }^{*}(T 2-T 3)\right) / V 3+D H R{ }^{*} r A /$ $\left(\right.$ RHO3 $\left.{ }^{*} C p\right)-\left(U 3{ }^{*} A 3 \_I N /\left(R_{H} H 3\right.\right.$ * $\left.\left.C_{p}^{*} V 3\right)\right)^{*}(T 3-T i n f 3)$

$T 3(0)=58 \# 48 \# 68$ cambio

$U 3=10$

$\# A 3=3.015936$

$A 3 \_I N=2{ }^{*} p i{ }^{*} R 3{ }^{*} h 3 \mathrm{RHO} 3=850$

$C p=4.184$

Tinf3 $=60 \# 50 \# 70$ cambio

$D H R=((H A C E T A T O+H A G U A)-$ $(H A C I D O+H A L C O H O L)) * 1000$

$H A G U A=-241.8$

HACETATO $=-392.226-7.0347 E-2$ * $T 3 K+3.460 E-5 * T 3 K \wedge 2$

$H A L C O H O L=-188.88-4.9823 E-2 *$

$T 3 K+2.0791 E-5 * T 3 K \wedge 2$

$H A C I D O=-422.584-4.8354 E-2 * T 3 K$ $+2.3337 E-5 * T 3 K^{\wedge} 2$

\# DHR = -14.437 calculado en función a la formula anterior

$r A=-k 1{ }^{*} C A{ }^{*} C B+(k 1 / K c){ }^{*} C C^{*}$ $C D r B=r A$

$r D=-r A r C=-r A$

$k 1=8.88 E 8{ }^{*} \exp (-7032.1 / T 3 K) / 60$ 160

$K c=5.2 * \exp ((-8000 / 1.978) *((1 /$ 298) $-(1 / T 3 K)))$

$T 3 K=T 3+273 N A=C A{ }^{*} V 3 N B=C B$

${ }^{*} V 3 N C=C C{ }^{*} V 3 N D=C D * V 3$

$F A=C A{ }^{*} V 3 p F B=C B * V 3 p F C=C C$ ${ }^{*} V 3 p F D=C D{ }^{*} V 3 p$

$N T=N A+N B+N C+N D Y A=N A /$ NT 
$Y B=N B / N T Y C=N C / N T Y D=N D$ / NT

$R E L 1=V 1 p / V 3 p R E L 2=V 2 p / V 3 p$

$R E L 3=(V 1 p+V 2 p) / V 3 p$

$X A=(C A E-C A) / C A E X B=(C B E-C B)$

/ $C B E$

REL_A1 $=$ h1 / h3 REL_A2 $=$ h2 / h3

$t(0)=0$

$t(f)=720$

\section{Resultados}

Se analiza el proceso de esterificación del ácido acético con metanol desde el punto de vista dinámico, lo cual permite hacer un análisis de los efectos de las variables en la operación del reactor semicontinuo. Los depósitos de los reactantes y productos tienen una geometría tal que el flujo de alimentación es variable, haciendo que la concentración de reactantes y productos varíen con el tiempo.

En la Figura 3 se observa la variación de la temperatura con el tiempo en cada uno de los tanques cilíndrico, cónico y esférico; se observa que luego de cierta inestabilidad al inicio del proceso, las temperaturas en los tres tanques, se estabilizan en valores constantes.

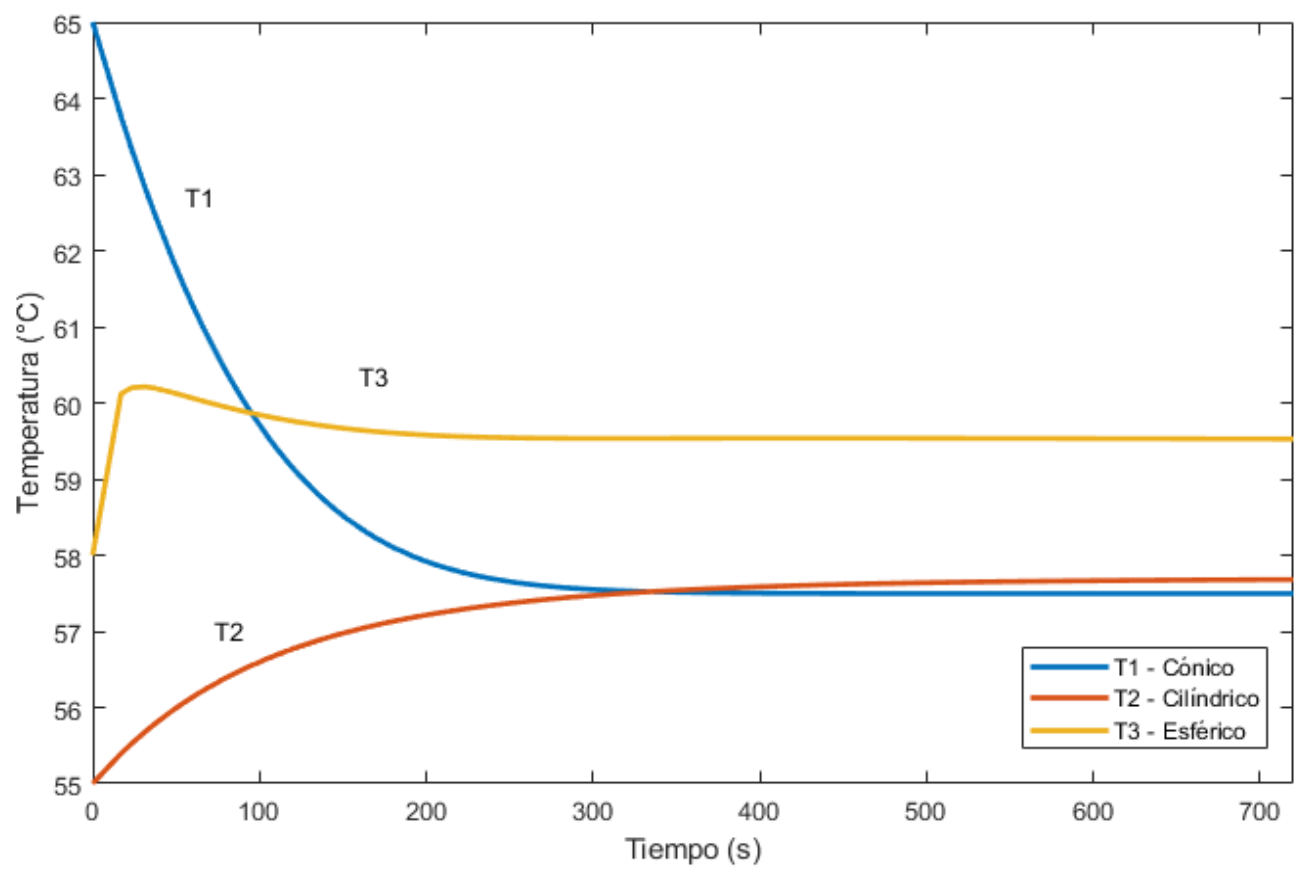

Figura 3. Temperatura en cada uno de los tanques vs tiempo

En la Figura 4 se observa que el fluido del tanque cónico disminuye su nivel al inicio del proceso, luego se estabiliza; esto indica que el flujo de entrada y el flujo de salida se igualan, mientras que en los tanques cilíndrico y esférico, para los datos suministrados, tienden a incrementarse. 


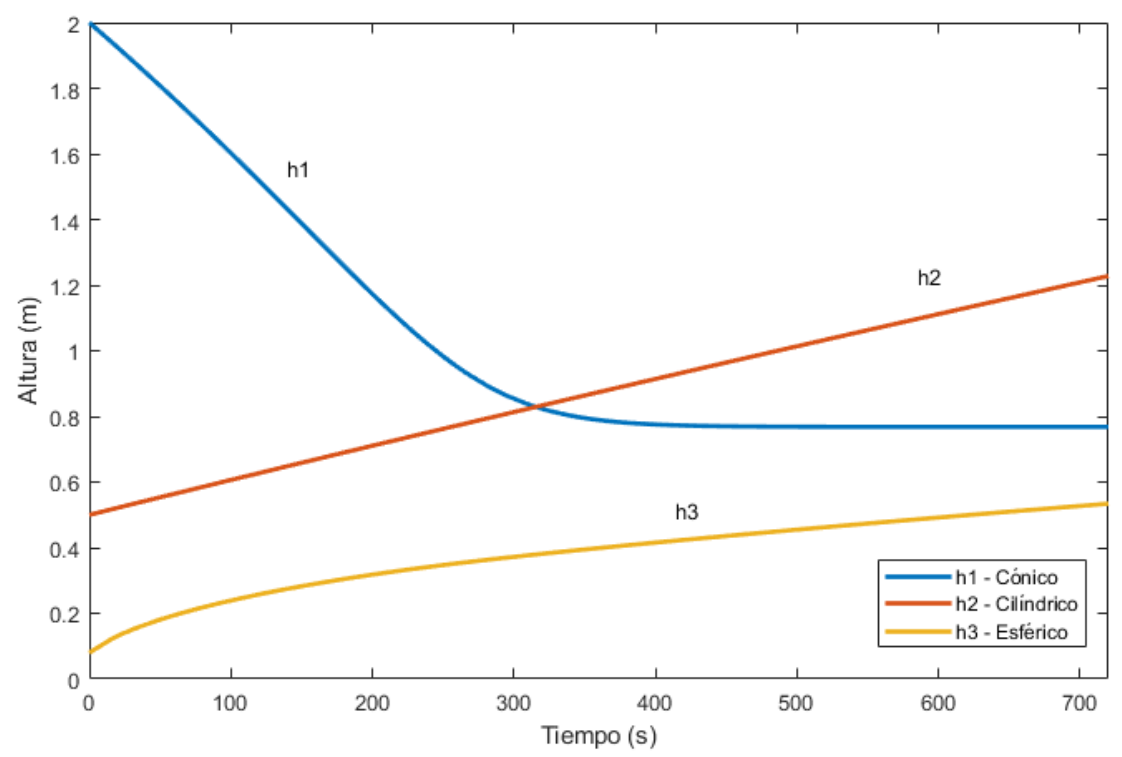

Figura 4. Variación de la altura de fluido en cada uno de los tanques vs tiempo

La Figura 5 muestra los perfiles temporales de concentración de cada uno de los componentes del proceso de esterificación del ácido acético con metanol; al principio hay un descenso rápido de la concentración de ácido acético y metanol por dilución; luego
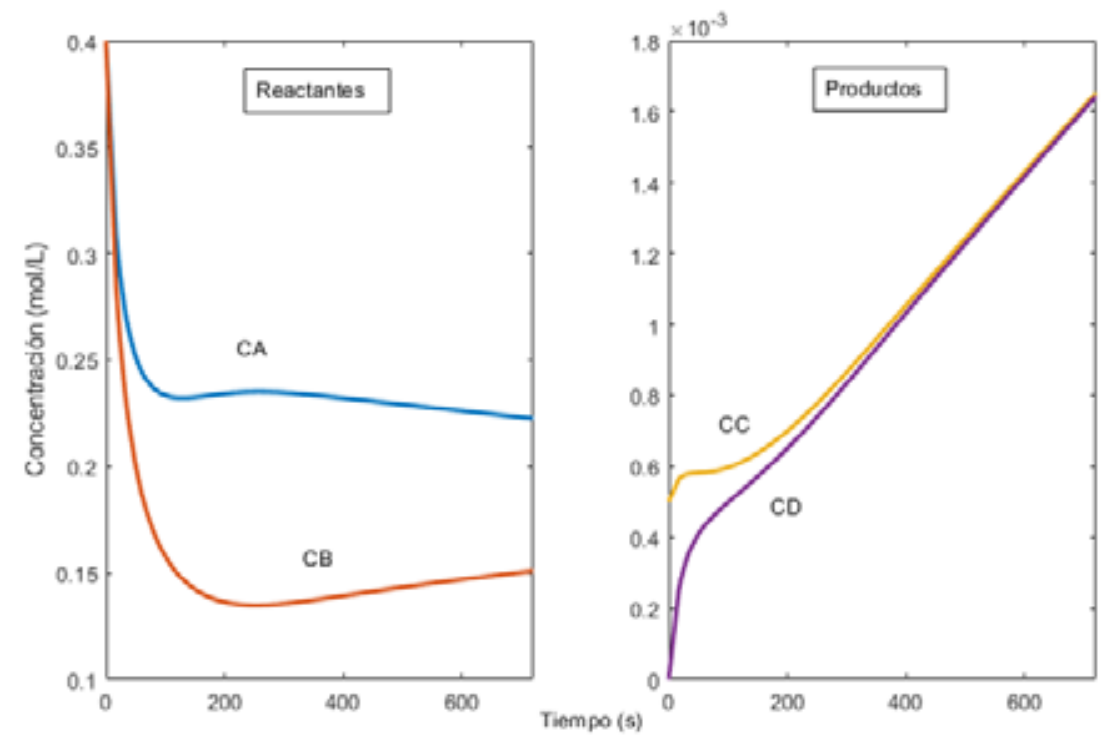

Figura 5. Concentración de los reactantes y productos en el reactor vs tiempo. comienza el proceso de reacción, haciendo que la concentración de ácido acético disminuya ligeramente y la de metanol se incremente por la mayor tasa de flujo. La concentración de agua y acetato de etilo se incremente sostenidamente; sin embargo, a un nivel bajo. 
En la Figura 6 se muestra el flujo molar de reactantes y productos del proceso de esterificación del ácido acético con metanol. Los flujos molares de los reactantes son más altos que los flujos

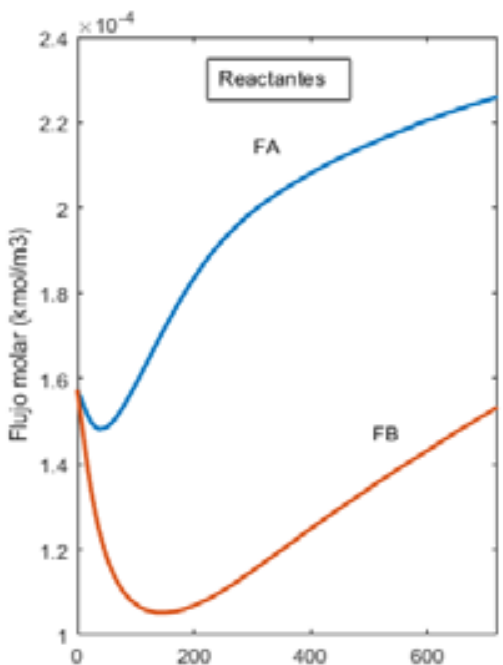

molares de los productos; esto debido a que el tiempo de residencia en el reactor es muy pequeño lo que impide que se llegue a condiciones cercanas al equilibrio.

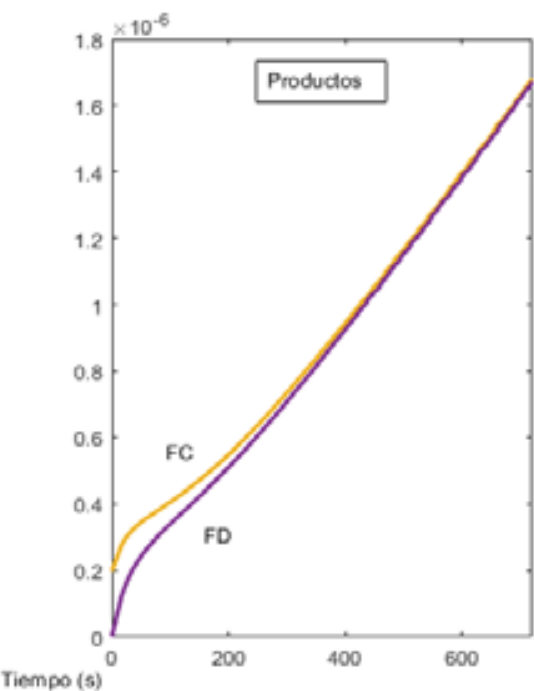

Figura 6. Flujo molar de los reactantes y productos a la salida del reactor vs tiempo.

En la Figura 7 se muestra la conversión del ácido acético y metanol en función al tiempo; al inicio se da un incremento rápido de la concentración; sin embargo, para tiempos más grandes, la conversión tiende a estabilizarse para ambos reactantes en niveles cercanos 0.4 para el ácido acético y 0.6 para el metanol.

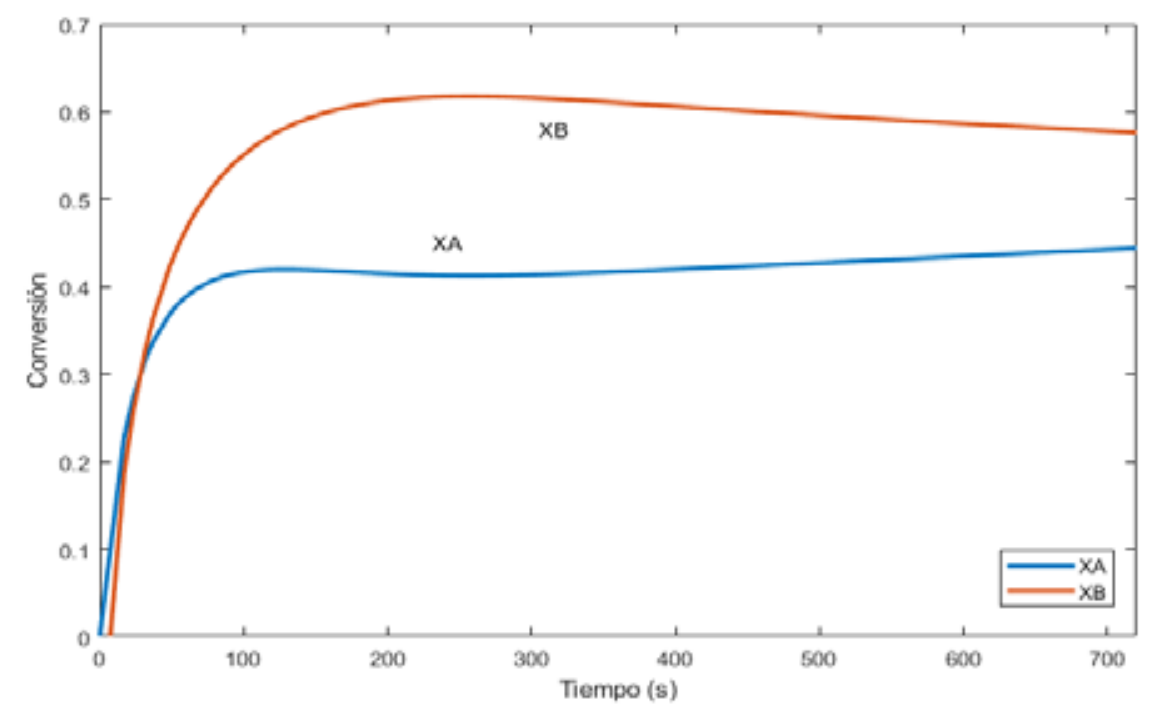

Figura 7. Conversión de los reactantes y productos a la salida del reactor vs tiempo. 
La Figura 8 muestra la evolución temporal de la composición molar; la fracción molar de acido acético tiene un comportamiento creciente decreciente con tendencia a estabilizarse, mientras que la fracción molar de metanol tiene un comportamiento decreciente creciente con tendencia a estabilizarse; las fracciones molares de acetato de metilo y agua tienen una tendencia creciente; sin embargo; los valores relativos de estos productos son mucho menores que la de los productos.
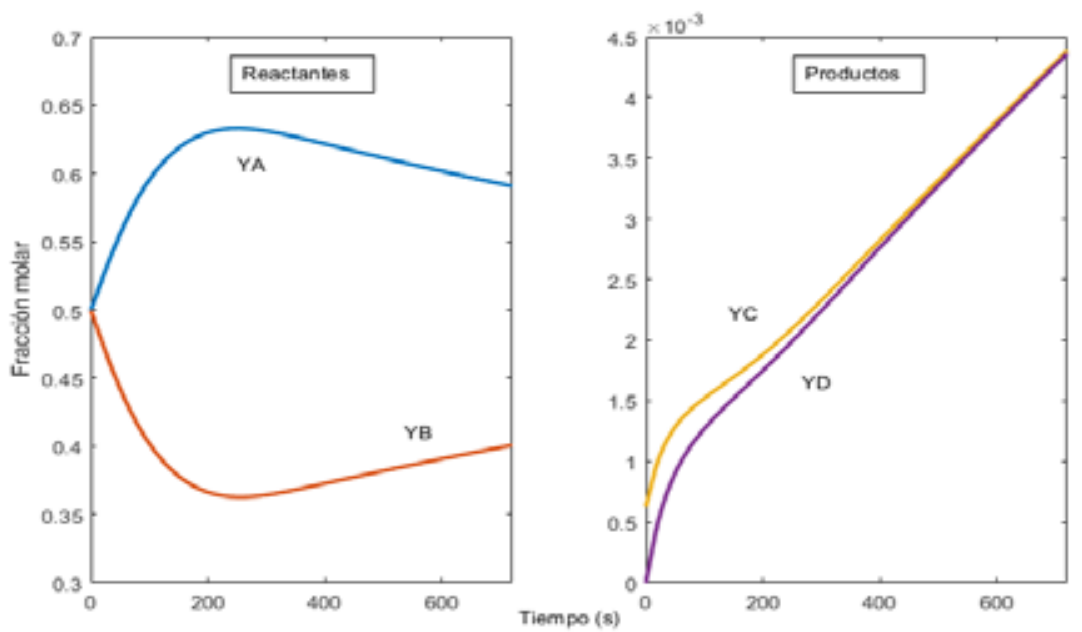

Figura 8. Fracción molar de los reactantes y productos a la salida del reactor vs tiempo.

En la Figura 9 se tiene las moles instantáneos de cada uno de los reactantes y productos en el reactor esférico, donde se observa un incremento de las moles totales de todos los componentes, debido a

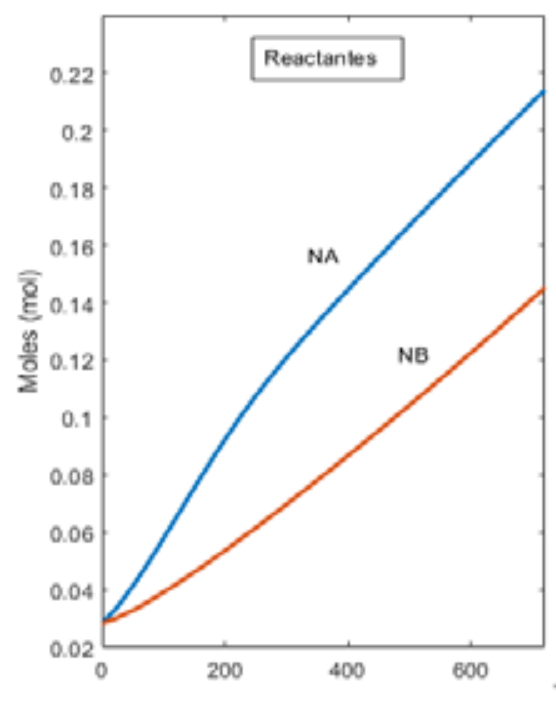

que la tasa de salida es menor que la tasa de entrada; asimismo las moles de producto son relativamente mucho menores que la de los reactantes; a su vez debido al corto tempo de residencia en el reactor

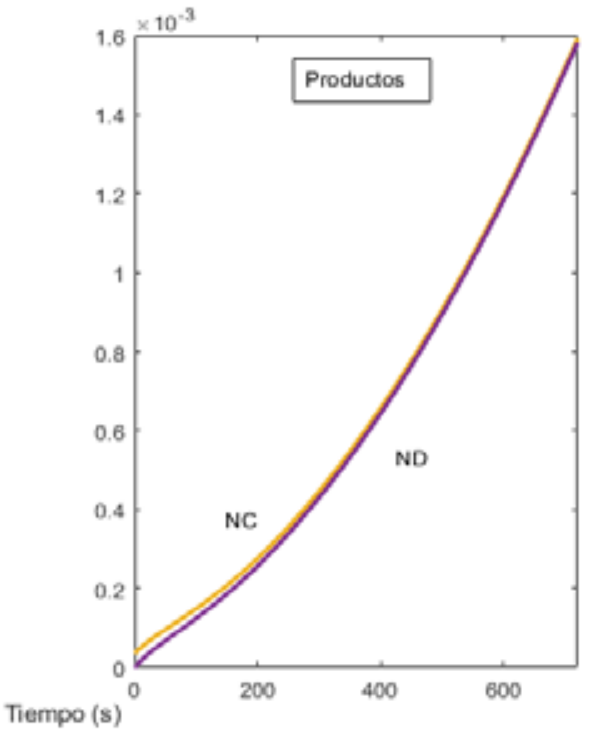

Figura 9. Moles de los reactantes y productos a la salida del reactor vs tiempo. 
En la Figura 9 se observa la relación de flujos volumétricos de los flujos de ingreso a cada uno de los tanques, respecto al flujo volumétrico de descarga; dicha relacion es decreciente en un principio, cuyo valor se estabiliza luego de unos 400 s. manteniéndose constante durante el proceso.

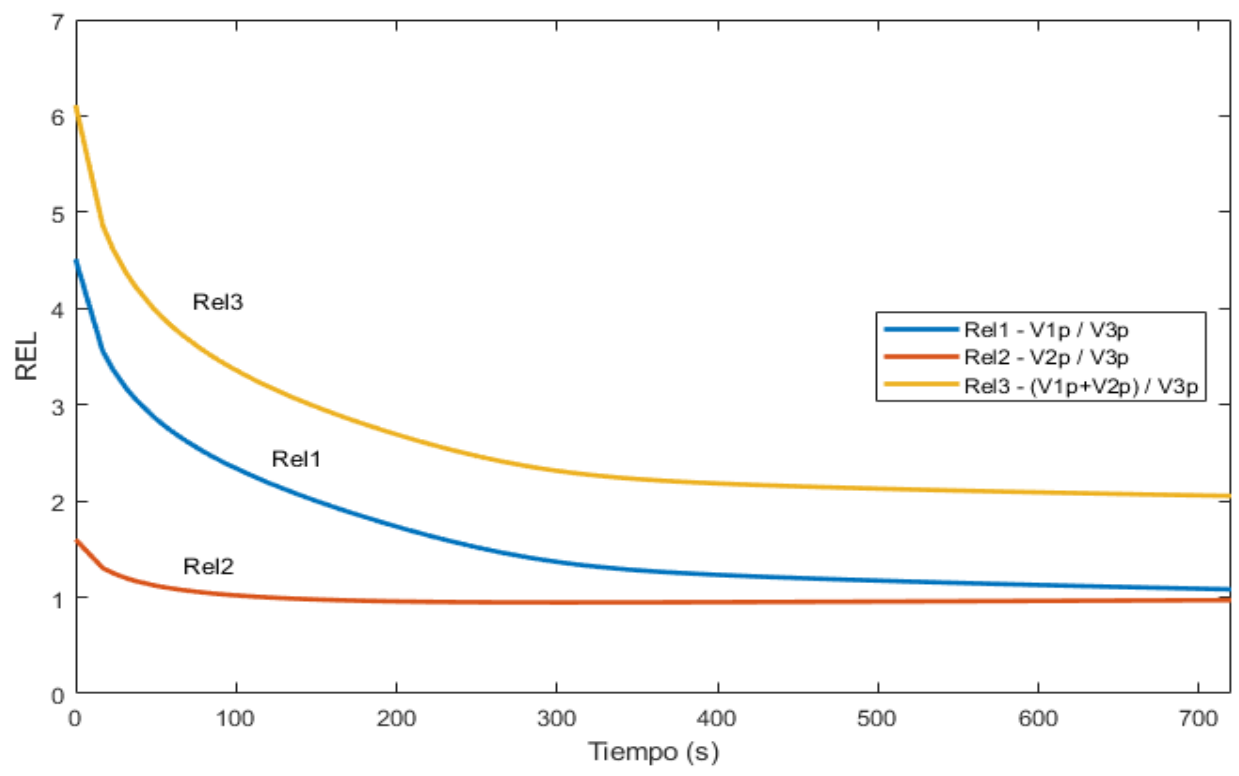

Figura 10. Relación de flujos volumétricos de entrada a los tanques respecto al flujo volumétrico de salida del reactor vs tiempo.

En la Figura 10 se muestra la relación de los flujos de entrada desde los tanques cónico y cilíndrico hacia el tanque esférico, respecto al flujo de salida; estos valores tienden a estabilizarse aproximadamente a $700 \mathrm{~s}$.

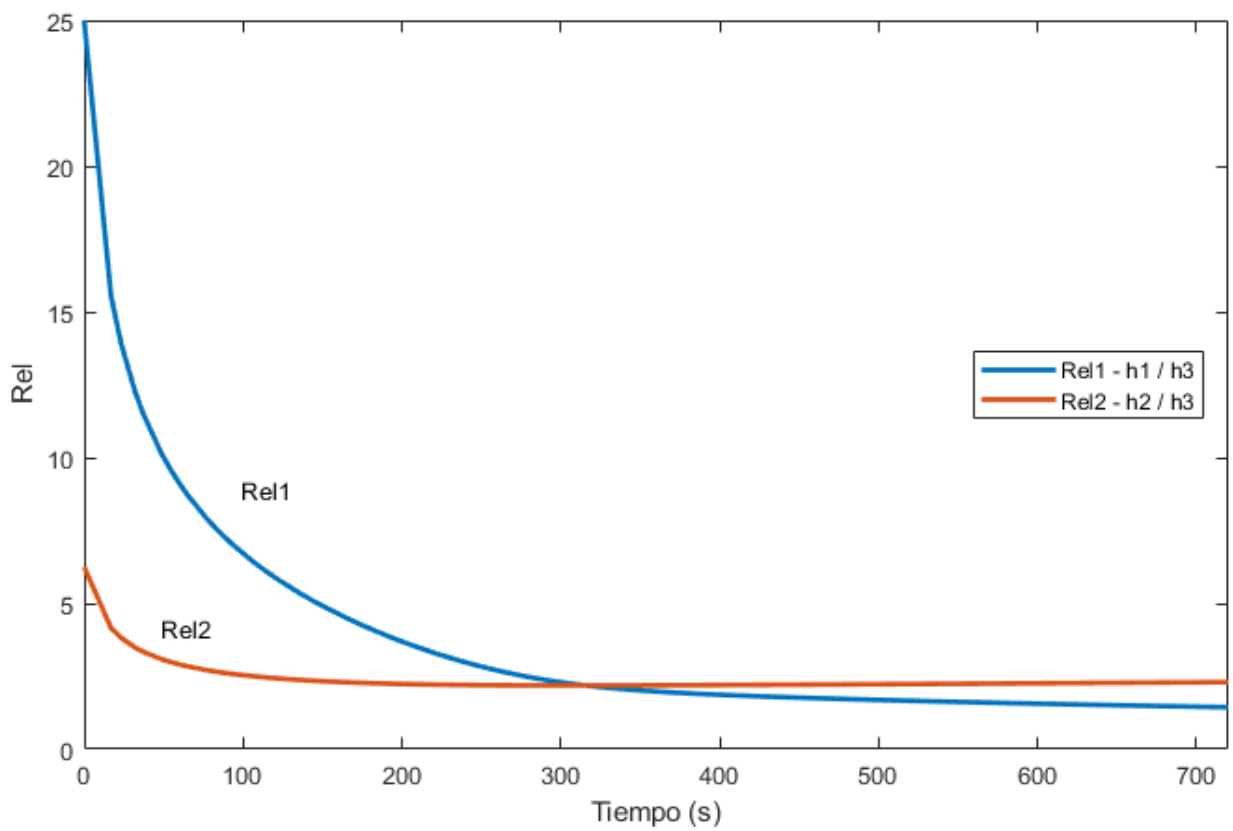

Figura 11. Relación de alturas de los fluidos contenidos en los tanques de ácido acético y metanol, respecto a la altura de la mezcla reaccionante vs tiempo 
La relación de las alturas instantánea de los fluidos contenidos en los tanques 1 y 2 respecto al tanque 3 se observa en la Figura 11; dicha relación es bastante variable al principio. En ambos casos, la altura de los fluidos en los tanques 1 y 2 es mucho mayor respecto al tanque 3 ; sin embargo, conforme pasa el tiempo, dicha relación tiende a ser constante, pues los datos de simulación permiten lograr esta condición de flujo cercana al equilibrio.

\section{Discusión de los resultados}

El flujo de descarga desde los tanques cónico y cilíndrico hacia el reactor depende de la geometría, el nivel inicial de los fluidos, el flujo de ingreso a los tanques y el flujo de descarga, que a su vez depende del diámetro de descarga; los niveles de temperatura en la descarga también son dependientes de las condiciones térmicas iniciales de los flujos, así como de la carga inicial.

Las condiciones finales de concentración de reactantes y productos, temperatura, flujo volumétrico, que se llevan a cabo en el reactor esférico, están en relación directa de las condiciones de flujo y temperatura proveniente de los tanques cónico y cilíndrico, así como de la condición térmica que se mantiene en el reactor esférico.

Con este programa desarrollado es posible simular una gran cantidad de posibilidades, variando las condiciones iniciales de operación en el reactor y en los tanques de alimentación, referido a las condiciones térmicas, concentraciones flujo neto de entrada y salida, etc.

Se estudió el efecto de las variables radio de salida $\mathrm{R} 03=[0.005 ; 0.01$; $0.015] \mathrm{m}$ y temperatura en la pared del reactor Tinf3 $=[50 ; 60 ; 70]{ }^{\circ} \mathrm{C}$ del reactor esférico frente a la conversión XA mediante un diseño factorial $2^{3}$, el cual queda representado en la siguiente tabla:

Tabla 1

Diseño factorial $2^{3}$ para $X A$

\begin{tabular}{ccc}
\hline R03 & Tinf3 & XA \\
\hline 0.01 & 60 & 0.3555 \\
0.015 & 70 & 0.3686 \\
0.005 & 60 & 0.3501 \\
0.005 & 50 & 0.3486 \\
0.01 & 70 & 0.3558 \\
0.015 & 50 & 0.3682 \\
0.015 & 60 & 0.3684 \\
0.01 & 50 & 0.3553 \\
0.005 & 70 & 0.3525 \\
\hline
\end{tabular}

Del análisis de varianza del diseño factorial de donde se obtuvo lo siguiente:

Tabla 2

\section{Análisis de varianza}

\begin{tabular}{crrrrr}
\hline Fuente & GL & SC Ajust. & MC Ajust. & Valor F & Valor $\mathrm{p}$ \\
\hline Modelo & 4 & 0.00052 & 0.00013 & 128.31 & 0.00 \\
Lineal & 4 & 0.00052 & 0.00013 & 128.31 & 0.00 \\
R03 & 2 & 0.000516 & 0.000258 & 254.7 & 0.00 \\
Tinf3 & 2 & 0.000004 & 0.000002 & 1.92 & 0.26 \\
Error & 4 & 0.000004 & 0.000001 & & \\
\hline Total & 8 & 0.000524 & & & \\
\hline
\end{tabular}

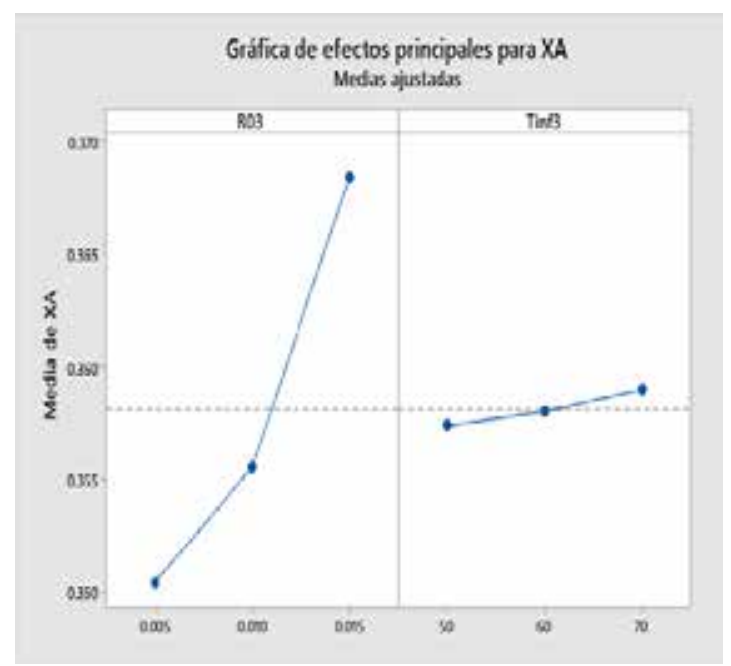

Figura 12. Gráfica de efectos principales 
Del análisis de varianza del diseño factorial se infiere al $95 \%$ de confianza que el aporte del radio de salida del reactor "R03" (valor $\mathrm{p}=0.00$ ) es significativo frente a la conversión XA y se maximiza la respuesta cuando se trabaja en el nivel alto $\mathrm{R} 03=0.015 \mathrm{~m}$. La influencia de la temperatura de la pared del reactor “Tinf3" resulta ser no significativa (valor $\mathrm{p}=0.26)$.

Así mismo, los supuestos del ANOVA son satisfechos: la normalidad de residuos y homogeneidad de varianzas, por lo que se confirma la validez de los resultados.

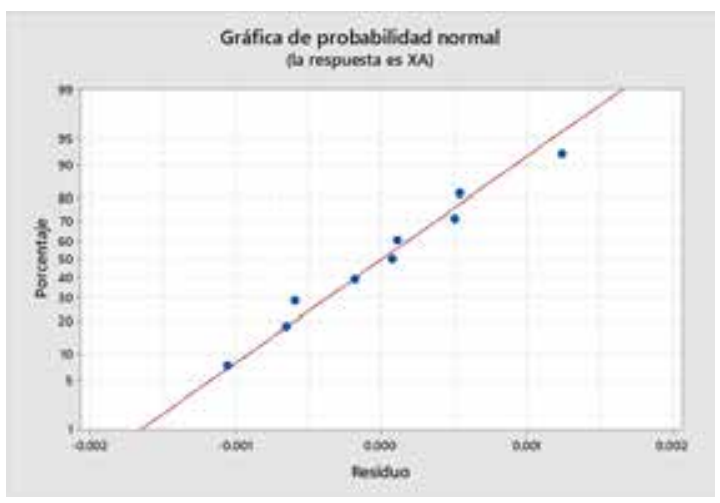

Figura 13. Normalidad de residuos

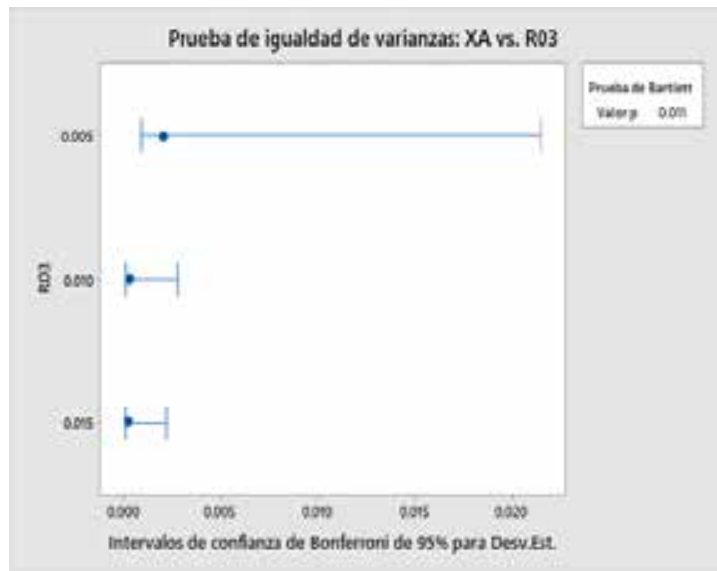

Figura 14. Homogeneidad de varianza R03

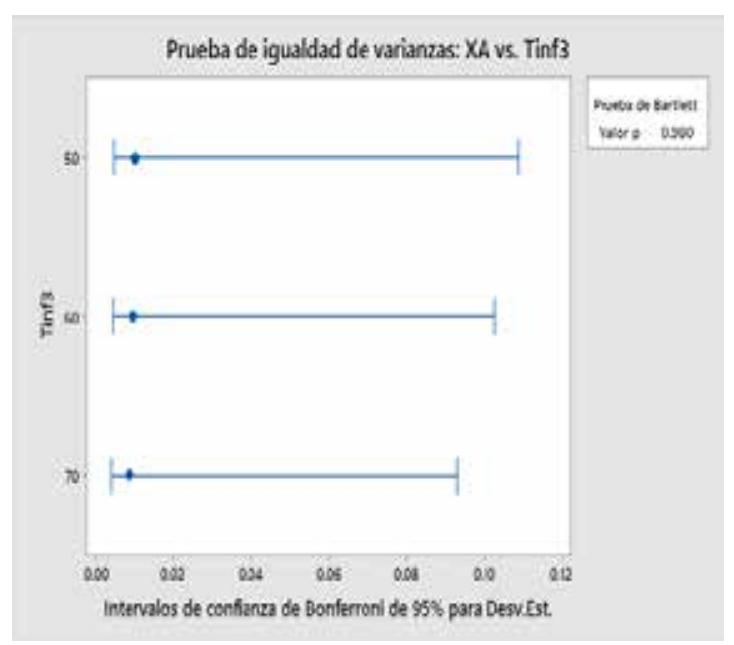

Figura 14. Homogeneidad de varianza Tinf3

\section{Referencias}

Aldás Arias, J.., \& Vivar Obregón, G,,. (2014). Diseño y construcción de un reactor semicontinuo para la obtención de acetato de sodio. Escuela de Ingeniería Química, Facultad de Ciencias. Escuela Superiior Politécnica de Chimborazo, Riobamba Ecuador.

Carrasco, L., (2018). Modelamiento de los fenómenos de transporte, $1 \mathrm{a}$. Ed. Edit. Macro SRL, Lima, Perú.
CRC Handbook of Chemistry and Physics, (1992), 73rd. Edition. Ed. David R. Lide. CRC Press. Boca Ratón, Florida.

Cutlip, M, Shacham, M., (2008). Resolución de problemas en ingeniería química y bioquímica con polymath, excel y matlab., 2a ed., Edit. Limusa Wiley, México.

Fogler, H. Scott. (2001). Elementos de ingeniería de las reacciones 
químicas. 3a ed. México: Pearson Educación, 2001.

Grau, D., (1999). Estudio del comportamiento de reactores discontinuos y semicontinuo: modelización y comprobación experimental; Universidad Politécnica de Cataluña, Barcelona, España.

Howard, Phillip H., and William M. Meylon. (1997). Handbook of Physical Properties of Organic Chemicals. CRC Press. Boca Raton, Florida. pag 595.

Huss, S., Chen, F., Malone, F., Doherty, M., (2003). Reactive distillation for methyl acetate production. Computer and Chemical Engineering Vol 27, 1855-1866.

Lux, S.; Winkler, T., Berger, G. and Siebenhofer, M. (2015), Graz University of Technology, Institute of Chemical Engineering and Environmental Technology, NAWI Graz, Inffeldgasse 25C, 8010 Graz, Austria.

Mallaiah, M. and Venkateswarlu, Ch. (2017). Kinetic behaviour of esterification of acetic acid with methanol over solid acid catalysts, Austin Chemical Publishing, Raju Institute of Technology, Narsapur, Telangana, India.

Ovando, V.,. (2007). Estudio teórico y experimental de la copolimerizacion de acetato de vinilo y acrilato de butilo en microemulsiones estabilizadas aniónica y estéricamente en procesos por lotes y semiconti- nuos. Centro de Investigación en Quiímica Aplicada, Saltillo, Mexico.

Perry's Chemical Engineering Handbook (1984), 6th Edition. Ed. Robert H. Perry and Don Green. McGrawHill Inc. New York.

Rohde, C; Marr, R., (2004), Investigation of methyl acetate production by reactive extraction. Department of Chemical Engineering and Environmental Technology University of Technology Graz Inffeldgasse 25, A-8010 Graz, Austria.

Ullmann's Encyclopedia of Industrial Chemistry, (1985). Ed. Wolfgang Gerhartz et. al. VCH Publishers. Deerfield Beach, Florida.

Xu, Z. P., and. Chuang, K., (1996). Kinetics of Acetic Acid Esterification over Ion Exchange Catalysts. The Canadian Journal of Chemical Engineering. Vol. 74 Aug: pp. 493-500.

Auquilla, L; Carrasco, L., (2017), "Modelamiento y simulacion del proceso de esterificacion del acido acetico con etanol en un reactor esferico", Tesis de Pre grado, Universidad Nacional del Callao, Peru.

Guerrero, M; Carrasco, L., (2017), "Modelamiento y simulacion del proceso de esterificacion del acido acetico con metanol en un reactor esferico", Tesis de Pre grado, Universidad Nacional del Callao, Peru 
Howard, Phillip H., and William M. Meylon. (1997). Handbook of Physical Properties of Organic Chemicals. CRC Press. Boca Raton, Florida. pag 595.

Huss, S., Chen, F., Malone, F., Doherty, M., (2003). Reactive distillation for methyl acetate production. Computer and Chemical Engineering Vol 27, 1855-1866.

Lux, S.; Winkler, T., Berger, G. and Siebenhofer, M. (2015), Graz University of Technology, Institute of Chemical Engineering a nd Environmental Technology, NAWI Graz, Inffeldgasse 25C, 8010 Graz, Austria.

Mallaiah, M. and Venkateswarlu, Ch. (2017). Kinetic behaviour of esterification of acetic acid with methanol over solid acid catalysts, Austin Chemical Publishing, Raju Institute of Technology, Narsapur, Telangana, India.

Ovando, V.,. (2007). Estudio teórico y experimental de la copolimerizacion de acetato de vinilo y acrilato de butilo en microemulsiones estabilizadas aniónica y estéricamente en procesos por lotes y semicontinuos. Centro de Investigación en Quiímica Aplicada, Saltillo, Mexico.

Perry's Chemical Engineering Handbook (1984), 6th Edition. Ed. Robert H. Perry and Don Green. McGrawHill Inc. New York.

Rohde, C; Marr, R., (2004), Investigation of methyl acetate production by reactive extraction. Department of Chemical Engineering and Environmental Technology University of Technology Graz Inffeldgasse 25, A-8010 Graz, Austria.

Ullmann's Encyclopedia of Industrial Chemistry, (1985). Ed. Wolfgang Gerhartz et. al. VCH Publishers. Deerfield Beach, Florida.

Xu, Z. P., and. Chuang, K., (1996). Kinetics of Acetic Acid Esterification over Ion Exchange Catalysts. The Canadian Journal of Chemical Engineering. Vol. 74 Aug: pp. 493-500. 
